\title{
Enantioselective Inhibition of Human Papillomavirus L1 Pentamer Formation by Chiral-Proline Modified Calix[4]arenes: Targeting the Protein Interface
}

\author{
Ding-Yi Fu, ${ }^{[a]}$ Tong Lu, ${ }^{[a]}$ Yu-Xue Liu, ${ }^{[a]}$ Fei Li, ${ }^{[a]}$ Mark I. Ogden, ${ }^{[b]}$ Ye Wang,${ }^{[c]}$ Yuqing \\ $\mathrm{Wu}^{*[\mathrm{a}]}$ and Mauro Mocerino ${ }^{*[\mathrm{~b}]}$ \\ [a] D.-Y. Fu, T. Lu, Y.-X. Liu, Prof. Dr. F. Li, Prof. Dr. Y. Wu \\ State Key Laboratory of Supramolecular Structure and Materials, Institute of Theoretical Chemistry \\ Jilin University \\ No. 2699 Qianjin Street, Changchun, 130012 (P.R. China) \\ E-mail: yqwu@jlu.edu.cn \\ [b] Prof. Dr. M. I. Ogden, Prof. Dr. M. Mocerino \\ Nanochemistry Research Institute and Department of Chemistry \\ Curtin University \\ GPO Box U 1987, Perth, 6845 (Australia) \\ E-mail: m.mocerino@curtin.edu.au. \\ [c] Dr. Y. Wang \\ School of Life Science, Jilin University \\ Changchun, 130012 (P.R. China) \\ Supporting information for this article is given via a link at the end of the document.
}

\begin{abstract}
Although current human papillomavirus (HPV) vaccines can protect well against infection, they are effective only for a limited number of subtypes. Coupled with the dilemma that no efficient prescription is currently clinically available for therapy, there is an urgent need to develop new anti-HPV agents. In the present study L- and D-Proline modified calix[4]arenes (Pro-C4A) were investigated to determine any differences in their effect on the assembly of HPV 16 L1 pentamer (L1-p). The mechanism of action using model peptides was investigated by MatrixAssisted Laser Desorption/lonization Time of Flight Mass Spectrometry (MALDI-TOF-MS) and Nuclear Magnetic Resonance (NMR) and revealed that the binding was targeting the basic residues at $L 1$ interface. This was also well supported by the trypsin digestion experiments and molecular simulations performed on the full-length L1. The large energy and morphology differences revealed by molecular simulations explain the binding disparity of L- and D-ProC4A to L1, and consequently the selective inhibition of them on L1-p formation. The present study opens a way to develop enantioselective and cost-effective inhibitors for L1-p formation, which might be used as a new kind of antiHPV agent and could be extended to other virus based on similar mechanisms.
\end{abstract}

\section{Introduction}

Human papillomaviruses (HPVs) are non-enveloped double-stranded DNA tumor viruse ${ }^{[1]}$ and consist of an icosahedral capsid, being composed of the major protein L1 and minor protein L2. ${ }^{[2]}$ High-risk HPV types have been shown to be the major etiological agents of cervical cancer ${ }^{[3,4]}$ Current vaccines for papillomavirus have been shown to be highly immunogenic, generally well tolerated and effective against limited number of phylogenetically related HPV types, but not against more distantly related subtypes. ${ }^{[5-8]}$ In particular, there is currently no clinically available efficient prescription for therapy. Therefore, there is an urgent need to develop new agents which are cost-effective and/or provide a greater broad-spectrum protection against HPV infections. In structure, the virion particles of HPV consist of 72 L1 capsomers and unknown numbers of the L2 minor protein, ${ }^{[9]}$ and each capsomer has distinct five-fold symmetry L1 pentamers (L1-p). ${ }^{[10]}$ Therefore, the L1 pentamer formation is the prerequisite of virion particle assembly, which renders it a key target to be monitored for virus control. The recent achievement of obtaining a stable glutathione S-transferase fusion L1 (GST-L1) monomer offered a unique strategy to monitor the kinetics of L1-p assembly. ${ }^{[11]}$ Later on, the watersoluble carboxylatopillar[5]arene (CP5A) and p-sulfonatocalix[4]arene (C4A) have been used to inhibit the HPV L1-p formation and finally prevent the assembly of virus particles. ${ }^{[12]}$ This opened a way to develop a new class of anti-HPV agents in targeting the key sites at the protein-protein interface. In an effort to develop more specific inhibitors on HPV assembly, new classes of chemical products, especially those with enantiomorphic functional groups, should be introduced.

Chirality is a prominent characteristic of biological molecules, and enantiomers of a chiral molecule often exhibit striking differences in terms of their physiological responses. ${ }^{[13]}$ Therefore, enantiomers have been widely applied to the various fields of science and technology, such as catalytic chemistry, biotechnology, and especially pharmaceutical science ${ }^{[14-16]}$ Consequently, numerous efforts have been devoted to the synthesis 
and application of artificial chiral host and/or receptors. ${ }^{[17,18]}$ Calixarenes are representative host molecules in supramolecular chemistry, ${ }^{[19]}$ and several chiral calixarene derivatives which incorporated chiral residues at either the wide or narrow rim, have been developed in recent years. ${ }^{[20-22]}$ However, very few reports have focused on exploring their potential pharmaceutical properties as anti-virus, although some of them have shown interesting levels of activity against bacteria, cancerous cells etc. ${ }^{[23}$ Therefore, in the present study the chiral proline-modified calix[4]arenes, L-Pro-C4A and D-Pro-C4A (Scheme 1), were used to investigate their effect on the assembly of HPV L1-p formation and any enantioselective inhibitions related to their chiral structural properties. So far, this is the first example of using chiral inhibitors against the pentamer formation of HPV L1. The intrinsic molecular mechanism of the enantioselective inhibition between them on the assembly of HPV 16 L1-p was explored using MALDI-TOF-MS, NMR and molecular docking simulation. In addition to the purpose of chiral recognition and discrimination, introducing amino acids at the rim of C4A is also expected to show higher matching and/or biocompatibility with proteins. ${ }^{[23]}$ Therefore, the present study opens a way to develop enantioselective inhibitors as a new class of anti-HPV agents by ceasing one key step of the virus life-cycle.
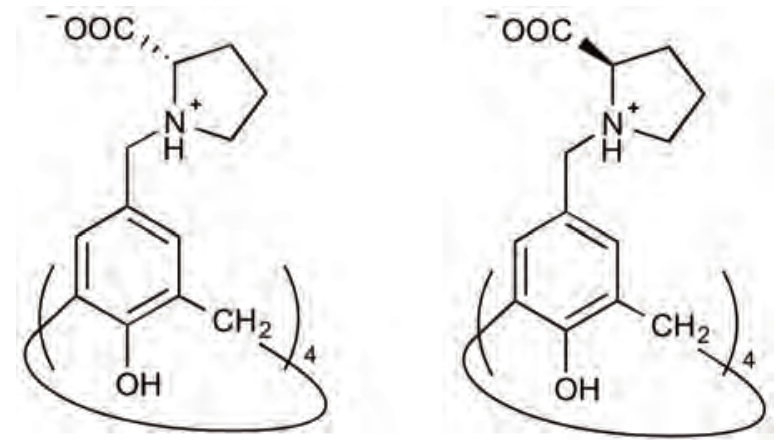

Scheme 1. The structures of the proline-modified calix[4]arene, L-Proline C4A (L-Pro-C4A) and D-Proline C4A (D-Pro-C4A).

\section{Results and Discussion}

In Vitro Inhibition of L-Pro-C4A on L1-p Assembly. The potency of L-Pro-C4A in inhibiting the L1-p assembly in vitro is firstly investigated by using static light scattering (SLS) measurement and the results are shown in Figure 1A. The monomer of GST-L1 can form L1-p after adding PreScission ${ }^{\text {TM }}$ Protease (PPase) to cleave the GST tag. ${ }^{[11]}$ As the size of pentamer is bigger than monomer, the formation of L1-p leads to an increase of the SLS intensity which is further used to monitor the kinetics of L1-p assembly. In the absence of L-Pro-C4A, the scattering intensity of the GST-L1 solution increased quickly with time after PPase addition for GST cleavage (Figure 1A, black), suggesting that the L1-p is formed steadily with the GST cleavage. However, in the presence of L-Pro-C4A, the increasing rate of intensity is markedly restrained, illustrating an obvious inhibition of L-Pro-C4A on L1-p formation. In addition, in the presence of more L-Pro-C4A the inhibition tendency is obviously strengthened. Finally, the presence of excess L-Pro-C4A to GST-L1 (600:1) almost fully suppresses the L1-p assembly, indicating the high potency of L-Pro-C4A on L1-p formation under these conditions.

To exclude the possibility that the intensity increase of SLS signal originated from protein aggregation in the SLS assay, the solution of L-Pro-C4A and GST-L1 (600:1) after measurement was then tested by using dynamic light scattering (DLS) measurement. The results showed a size distribution of hydrodynamic diameter at $\sim 8.5 \mathrm{~nm}$ (Figure 1B, upper), being essentially the mixture of GST tag and L1 monomer (L1-m) rather than any aggregation of protein. However, without L-Pro-C4A participation, the PPase cleaved GST-L1 showed a distribution of hydrodynamic diameter centered at $\sim 15.7 \mathrm{~nm}$ (Figure 1B, bottom). This is the expected size of the L1- $p,{ }^{[24]}$ confirming the SLS intensity increase positively correlates with the L1-p formation. 


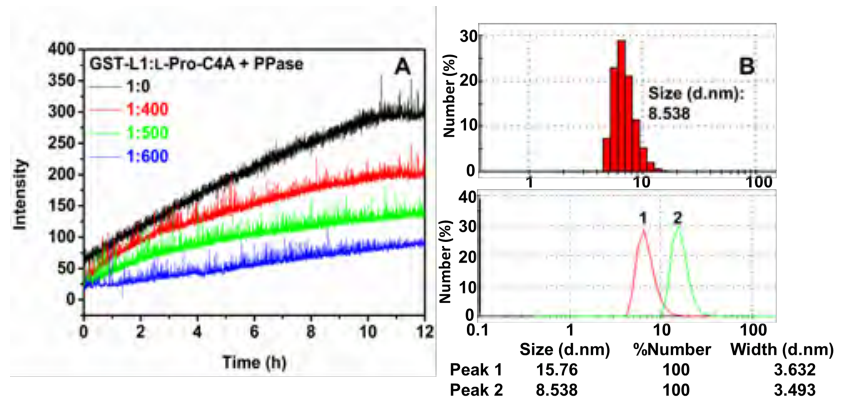

Figure 1. (A) Time-dependent monitoring of the self-assembly of L1-p from GST-L1 monomers (1.25 nM), under different concentrations of L-Pro-C4A, by SLS after PPase addition. (B) DLS profiles, showing the hydrodynamic diameter distribution of the protein after incubating with PPase in the absence (peak 1) and presence (peak 2) of L-Pro-C4A (1:600).

Then the inhibition efficiency of L-Pro-C4A on HPV 16 L1-p assembly was assayed directly by using sizeexclusion chromatography (SEC). The GST-L1 was first pre-incubated with different quantities of L-Pro-C4A (0 to 600 equivalents) in solution overnight. Then PPase was added before the solution was injected onto gelfiltration columns and subjected to the fast protein liquid chromatography (FPLC). The obtained elution profiles are shown and compared in Figure 2A. Without the pre-incubation with L-Pro-C4A, the FPLC elution profile shows an intense peak at $\sim 265 \mathrm{kDa}$ and a weak peak at $\sim 53 \mathrm{kDa}$, being attributed to the position of HPV L1-p and L1-m, respectively, based on the previous reports. ${ }^{[1,25]}$ Treatments with different molar ratios of L-ProC4A to GST-L1 endow FPLC elution profiles with weaker peaks for L1-p but stronger ones for L1-m. The conversion is illustrated more clearly as the intensity ratio of L1-p to L1-m, which correlates negatively with the L-Pro-C4A concentrations in solution (Figure S1). More precisely in Figure 2B, the percentage of L1-p formation inhibited by L-Pro-C4A is shown in a dose-dependent manner with a half-inhibition concentration $\left(\mathrm{IC}_{50}\right)$ of $2.21 \mathrm{mM}$, in preventing the L1-p assembly from a solution containing $1.25 \mathrm{nM}$ GST-L1. Therefore, the inhibition of L1-p assembly by L-Pro-C4A in vitro is shown to be reliable and efficient after three repeated measurements.
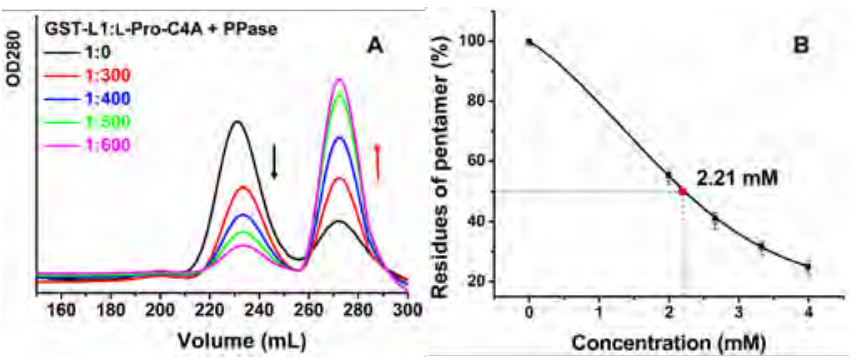

Figure 2. (A) Examinations of the L1-p formation by SEC. The molar ratios of L-Pro-C4A to GST-L1 are changed from 0:1 to 600:1, respectively. (B) The curve illustrates the L1-p yield under the inhibition of L-Pro-C4A and the corresponding $I C_{50}$.

In Vitro Inhibition of D-Pro-C4A on L1-p Assembly. To investigate how the enantiomer influences on L1-p assembly, the upper rim of calix[4]arene was functionalized with D-Proline to produce D-Pro-C4A by using the reported method. ${ }^{[26]}$ Both of the enantiomers are firstly characterized by using circular dichroism (CD) spectra (Figure S2), which confirmed their chiral structures. Then the D-Pro-C4A was used to investigate the inhibition kinetics on L1-p assembly by using SLS and the results are shown in Figure 3A. The efficiencies were found to be similar to those of the L-Pro-C4A, but being somewhat less effective. Although the inhibition tendency increased with the addition of more D-Pro-C4A, it was obviously less efficient than that of L-Pro-C4A. As shown, in the presence of high molar ratio of D-Pro-C4A to GST-L1 (600:1), the SLS intensity was restrained only $55.1 \%$ after 12 hours incubation; while $72.3 \%$ was inhibited by L-Pro-C4A at the identical condition. The comparisons of the concentration-dependent SLS plots of L1-p assembly in Figure 3B clearly illustrate lower inhibition efficiencies induced by D-Pro-C4A than L-Pro-C4A. Especially, in terms of the kinetics, L-Pro-C4A inhibited the L1-p assembly once it was added at the start of the SLS monitoring (Figure 1A), while there was almost no inhibition on L1-p formation during the first two hours after low molar ratio of D-Pro-C4A $(<500: 1)$ was added (Figure $3 \mathrm{~A}$ ). 
The inhibition efficiency of D-Pro-C4A against HPV 16 L1-p assembly was also detected by using SEC. The GST-L1 was treated at the identical conditions for the L-Pro-C4A as described, but different results were obtained. The FPLC elution profiles (Figure S3A) show that as the concentration of D-Pro-C4A increased, the peak for L1-p decreased while that for L1-m increased in intensity. The rate of L1-p assembly was also inhibited by D-Pro-C4A in a dose-dependent manner, but the $\mathrm{IC}_{50}$ was evaluated to be $3.06 \mathrm{mM}$ to prevent the L1-p formation from a solution of $1.25 \mathrm{nM}$ GST-L1 (Figure S3B). Therefore, the inhibition of L1-p formation by $\mathrm{D}-$ Pro-C4A in vitro was relatively less than that of L-Pro-C4A, and the intrinsic mechanism of the difference will be discussed in the following.
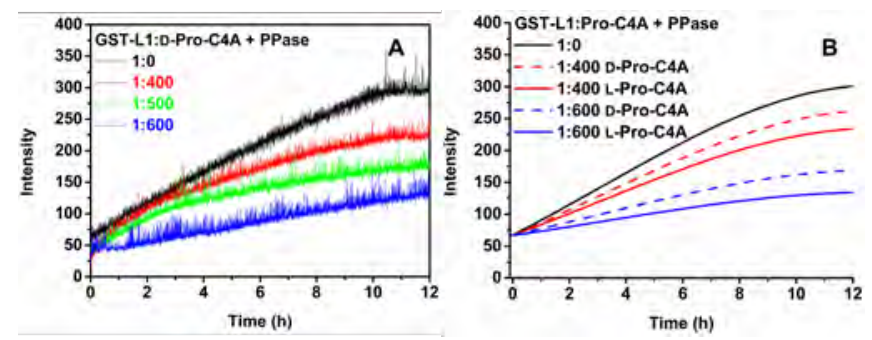

Figure 3. (A) Monitoring of the assembly of L1-pentamers from GST-L1 monomers (1.25 nM) with different concentrations of D-ProC4A by time-dependent static light scattering (SLS), which are recorded after the addition of the PPase for GST cleavage. (B) Comparison of the concentration-dependent static light scattering (SLS) plots of L1-p formation as monitored by the D-Pro-C4A (dashed line) and L-Pro-C4A (connected line).

A mixture of diastereoisomers prepared by the Mannich reaction of $\mathrm{C} 4 \mathrm{~A}$ with racemic proline was found to have no inhibition on HPV 16 L1-p formation at high molar ratios (300:1 and 500:1). However, it was interesting to observe that slight inhibition on L1-p formation was observed at a lower molar ratio $(80: 1)$ (Figure S4), although the inhibitory effect was not very expeditious. That is, low concentrations of the diastereomeric mixture show a slight inhibition on L1-p formation while the higher concentrations did not. Such a unique result might be related to the tendency of diastereoisomers to self-assemble. ${ }^{27-29}$ It was reported that the racemate appears to form linked spheroidal structures at high concentration (5.6 mM). The self-assembly of Pro-C4A will make it very difficult to encapsulate the residue at the protein interface and finally show almost no inhibition on L1-p formation. Therefore, such results confirm that Pro-C4A indeed binds to the key sites at L1 interface, which efficiently stop the L1 interaction in forming L1-p.

Clarification of Pro-C4A Binding Site at L1 Interface. The intrinsic mechanism of the specific reorganization, at least the exact binding site of Pro-C4A on L1 was initially explored. A previous gelation study showed that three basic amino acids (arginine, histidine and lysine) could non-covalently interact with the L-Pro-C4A in micelles, ${ }^{[30]}$ and therefore they were suggested to be the first candidate group to bind with Pro-C4A. As multibinding sites might be involved in HPV L1, a short peptide, with only one positive arginine residue, DLDQFPLGR (pep 9) originated from helix 5 of HPV 16L1, was used as a simplified model to clarify the exact binding sites of Pro-C4A with L1. NMR spectroscopy is a powerful and commonly used method to directly investigate the interaction of two molecules, especially to determine the exact binding sites. ${ }^{[31]}$ The amide region of ${ }^{1} \mathrm{H}$ NMR spectra of pep 9 in binding with L-Pro-C4A at different molar ratio are shown in Figure 4. Two dimensional Total Correlation Spectroscopy (TOCSY) was used to identify the spin system of an individual amino acid residue, while Nuclear Overhauser Effect Spectroscopy (NOESY) was used to establish backbone sequential connectivity (Figure S5). The assignments of the ${ }^{1} \mathrm{H}$ resonances were then determined based on the 2D NMR spectra (Figure S5 and Table S1) and the standard methods. ${ }^{[2]}$ This made it possible to analyse the ${ }^{1} \mathrm{H}$ NMR spectra of the peptide in the absence and presence of different amounts of L-Pro-C4A (Figure 4) and D-Pro-C4A (Figure S6). The chemical shifts of the backbone NH protons in the guanidinium of arginine are markedly affected upon addition of Pro-C4A, indicating the high possibility of Arg binding with Pro- C4A. The binding of Pro-C4A to L1 results in up-field shifts in $\mathrm{NH}$ proton resonances, while the resonances of $\mathrm{H} \alpha$ proton shift slightly down-field. That means that the cavity of C4A provides a shielding environment for guest protons, which induces an up-field ${ }^{1} \mathrm{H}$ NMR chemical shift change $\left(\Delta \delta_{\text {lim }}=\delta_{\text {bound }}-\delta_{\text {free }}\right)$; while down-field shifts are observed for guest protons outside of the cavity adjacent to the polar portals. The limiting value of $\Delta \delta_{\text {lim }}$ for the $\mathrm{NH}$ protons on arginine $(-0.271)$ is bigger than those of the $\mathrm{H} \alpha(-0.083)$ bound to Pro-C4A, suggesting that the $-\mathrm{NH}^{2+}$ group interacts with the cavity of the host. 


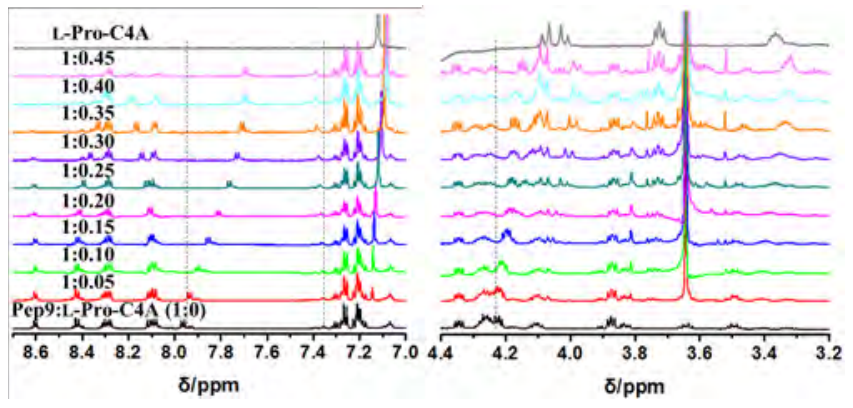

Figure 4. ${ }^{1} \mathrm{H}$ NMR spectra of $1 \mathrm{mM}$ HPV L1 pep 9, with L-Pro-C4A at the molar ratio (peptide/L-Pro-C4A) from 1:0.05 to 1:0.45 and that of L-Pro-C4A itself, which are all measured in $90 \% \mathrm{H}_{2} \mathrm{O} / 10 \% \mathrm{D}_{2} \mathrm{O}$.

The binding sites of Pro-C4A at GST-L1 interface were subsequently confirmed by using the MALDI-TOF-MS (Figure 5) of a longer 14 peptide, DLDQFPLGRKFLLQ (pep14), originated from HPV 16L1. The intense peak at $\mathrm{m} / \mathrm{z}=1691.8 \mathrm{Da}$ is essentially assigned for a pep 14 ; while the appearance of a peak at $\mathrm{m} / \mathrm{z}=3557.4 \mathrm{Da}$ corresponds to the molecular weight of one pep14 and two Pro-C4A molecules, illustrating a peptide could bind to two Pro-C4A firmly. As two cationic residues ( $K$ and $R$ ) are included in one peptide, this suggests the Pro-C4A does indeed bind to the positive residues such as $K$ and $R$. In particular, the peak intensity difference observed between L-Pro-C4A and D-Pro-C4A in binding with pep14 (insert of Figure 5) reveals that the L-ProC4A could bind to the peptide more tightly than D-Pro-C4A.

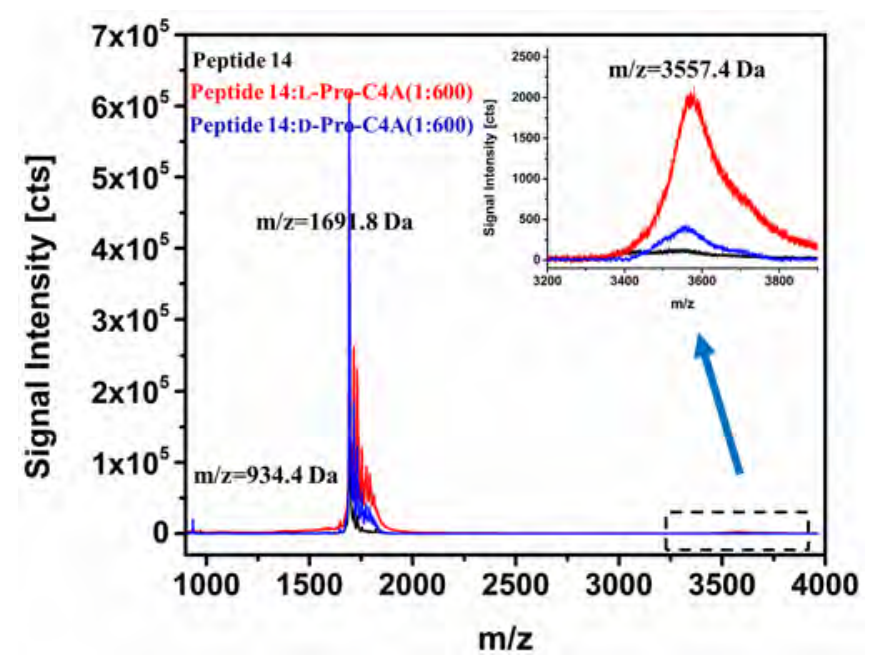

Figure 5. MALDI-TOF-MS of peptide 14 (black) and it in complexion with L-Pro-C4A (red) and D-Pro-C4A (blue), respectively.

We also carried out an enzymatic digestion experiment by using trypsin to further confirm whether Pro-C4A was truly bound at the positive residues of full length GST-L1. It is well known that trypsin exclusively cleaves the arginine $(R)$ and lysine $(K)$ residues in proteins. ${ }^{[3]}$ Taking GST-L1 as an example, if the sites for enzyme digestion and Pro-C4A binding were the same, the pre-incubation of GST-L1 with Pro-C4A would prevent the digestion of GST-L1 by trypsin; otherwise, there would be no interference on the digestion. SDSPolyacrylamide Gel Electrophoresis (SDS-PAGE) was used as an assay to test this hypothesis. The results showed that without Pro-C4A participation the GST-L1 was almost degraded completely by trypsin in 7 min after the addition (Figure 6, panel 2); whereas in the presence of Pro-C4A, the degree of hydrolysis was clearly reduced (Figure 6, panel 3,4 ). These results indicate that, when allowed to interact with GST-L1, ProC4A indeed binds to the positively charged residues of Lys and Arg and therefore protects it from trypsin degradation. Such results are similar to previous reports for other proteins, ${ }^{[33,24]}$ which supports our conclusion here. In addition, the comparison of gray values in panel 3 and 4 indicate that L-Pro-C4A provides a better protection than D-Pro-C4A (Figure 6), which can be attributed to the enantiomeric differences in binding to GST-L1. Therefore, the comparison in Figure 5 shows that a peptide could bind to two Pro-C4A molecules at the same time, confirming again that the binding between them is targeting Arg and Lys. In particular, the different peak intensities for the complexes indicate that L-Pro-C4A can bind the peptide more tightly than D- 
Pro-C4A. That is, the cavity of L-Pro-C4A and D-Pro-C4A could recognize and bind with the basic residues of GST-L1, but the orientation of chiral proline moieties determine the binding strength of Pro-C4A to the protein, which will be clarified in detail by the following molecular simulations.

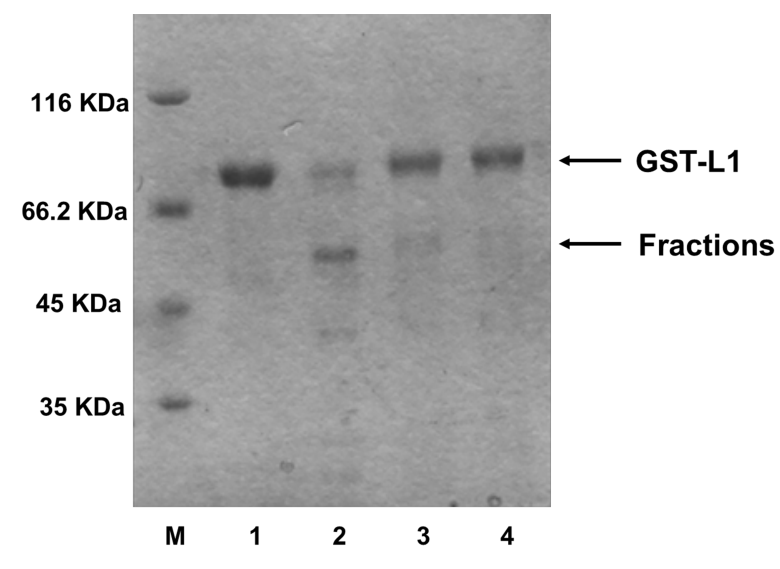

Figure 6. SDS-PAGE analysis shows the effect of Pro-C4A on GST-L1 digestion by trypsin. Panel 1: GST-L1 itself; and Panel 2: GST-L1+Trypsin (2.5 mg mL $)$, Panel 3: (GST-L1+D-Pro-C4A)+Trypsin (2.5 mg mL $\left.{ }^{-1}\right)$, Panel 4: (GST-L1+L-Pro-C4A)+Trypsin (2.5 mg $\left.\mathrm{mL}^{-1}\right)$, after 7 min incubation, respectively.

Molecular Docking Simulation to Reveal the Intrinsic Mechanism of the Enantioselective Inhibition of Pro-C4A on L1-p Formation. Finally, molecular docking simulation was performed to investigate in more detail how L-Pro-C4A and D-Pro-C4A bind to GST-L1 differently. AutoDock is used to estimate the potential interaction and the conformation of the protein-ligand complex. There are 14 basic residues, 7 Arg and 7 Lys, involved in each L1 monomer, these being the possible binding sites to Pro-C4A based on the above results. Therefore, they were used as the possible binding targets for the docking performance. The predicted complex was optimized and ranked according to the empirical scoring function, Screen Score, which estimates the binding free energy $(\Delta \mathrm{E})$. The results in Table S2 show that for each possible site in $\mathrm{L} 1$ the $\Delta \mathrm{E}$ to L-Pro-C4A is lower than that to D-Pro-C4A, finally resulting in a total energy difference of $11.24 \mathrm{kcal} \mathrm{mol}^{-1}$ between two complexes. In particular, significant energy difference $\left(\Delta \mathrm{E}_{\mathrm{D}}-\Delta \mathrm{E}_{\mathrm{L}}>1.0 \mathrm{kcal} \mathrm{mol}^{-1}\right)$ was observed at the sites of R41, K217, R251, R252 and R258, respectively, which contributed greatly to the differentiation between L- and D-Pro-C4A. In addition, there were 6 basic residues at the interface of each L1 monomer forming the pentamer, which contribute total energy differences of $-5.06 \mathrm{kcal} \mathrm{mol}^{-1}$ in binding with $\mathrm{L}$ - and $\mathrm{D}$ Pro-C4A (Table 1). The most significant energy differences $\left(\Delta E_{D}-\Delta E_{L}>1.0 \mathrm{kcal} \mathrm{mol}^{-1}\right)$ are observed at the sites of R41 and R258, so these are analyzed in detail below.

Table 1. The estimated free energy and each difference of basic residues at interface of L1 in binding with L- and D-Pro-C4A, respectively.

\begin{tabular}{|c|c|c|c|}
\hline Residue & $\begin{array}{c}\text { +L-Pro-C4A } \\
\text { Energy }\left(\mathrm{kcal} \mathrm{mol}^{-1}\right)\end{array}$ & $\begin{array}{l}+\mathrm{D}-\text { Pro-C4A } \\
\text { Energy }\left(\mathrm{kcal} \mathrm{mol}^{-1}\right)\end{array}$ & $\begin{array}{c}\text { Differences } \\
\text { Energy }\left(\mathrm{kcal} \mathrm{mol}^{-1}\right)\end{array}$ \\
\hline $\mathrm{R} 41$ & -4.95 & -3.90 & -1.05 \\
\hline K125 & -6.26 & -5.52 & -0.74 \\
\hline R144 & -5.86 & -5.26 & -0.60 \\
\hline R258 & -6.15 & -4.88 & -1.27 \\
\hline K356 & -3.79 & -2.81 & -0.98 \\
\hline K466 & -3.45 & -3.03 & -0.42 \\
\hline Total & -30.46 & -25.40 & -5.06 \\
\hline
\end{tabular}


Large differences were also observed in morphology between L- and D-Pro-C4A in binding to L1. For example, the residue of R41 could form hydrogen bonds both with L- and D-Pro-C4A, but the strength of hydrogen bond in L-Pro-C4A $(1.72 \AA)$ is relatively stronger than that in D-Pro-C4A $(1.94 \AA)$ according to their distances (Figure S7). In addition, the residue of R258 showed a greater difference than R41 in forming hydrogen bonds with LPro-C4A and D-Pro-C4A, reflected by the bigger gap of hydrogen bonds distances (1.59 $\AA$ vs $2.05 \AA$, Figure S8). Furthermore, L-Pro-C4A can bind more closely to the residue of Lys 54 (Figure 7) and Lys 467 (Figure S9) in forming stronger multi-hydrogen bonds in comparison to the D-Pro-C4A. Consequently, L-Pro-C4A could interact with Lys 54 and form several stronger hydrogen bonds with it and the neighboring residues. The large energy and morphology differences observed here are consistent with the binding disparity of L- and D-ProC4A to L1, and consequently the selective inhibition of them to L1-p formation. Therefore, both the amino acid functional groups and the cavity of the calix[4]arene cooperate well in chiral recognition to HPV L1 monomer, and subsequently lead to the enantioselective inhibition on L1-p formation.

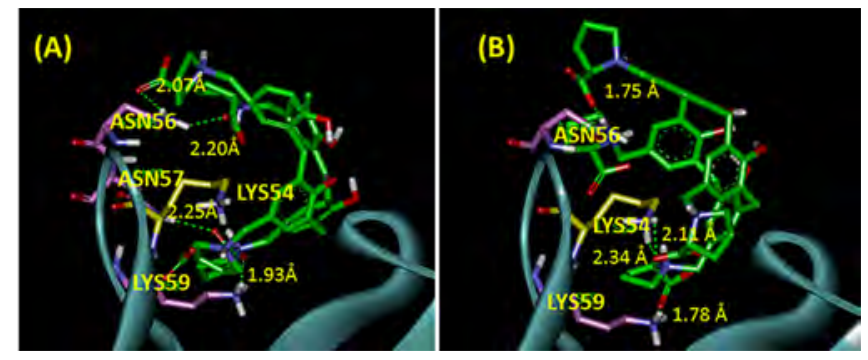

Figure 7. Hydrogen bonds formed between the target residue of Lys 54 in HPV 16 L1 and (A) D-Pro-C4A (B) L-Pro-C4A, respectively, the distance of hydrogen-bonds were represented as green dashed lines.

\section{Conclusions}

A pair of chiral calix[4]arenes were applied to recognize the capsid protein of HPV 16 L1 in this study. Most notably, they show enantioselective inhibition of L1-p formation. Both the MALDI-TOF-MS and NMR of these two C4A derivatives in complexing with model peptides reveal that the binding targeted the basic residues at the L1 interface. Subsequent trypsin digestion experiments and molecular docking simulations for the fulllength L1 support this conclusion. The present study opens a way to develop enantioselective and costeffective inhibitors as new anti-HPV agents, which could be extended to other viruses based on the identical molecular mechanism. Currently, investigations on novel C4A compounds with more negative charges at the wider rim are ongoing, which are expected to improve the binding affinity to GST-L1 and especially the enantioselective inhibition of L1-p formation.

Electronic Supplementary Information (ESI) available: Experimental procedures, plots of $S p / S m, C D$ spectra of pro-C4A, FPLC elution profiles of D-Pro-C4A and D/L-Pro-C4A on L1-p formation, ${ }^{1} \mathrm{H}$ and $2 \mathrm{D}$ TOCSY NMR spectra of Pro-C4A and the peptide model interaction, and Autodock simulation results.

\section{Acknowledgements}

The present work was supported by the NSFC (Nos. 91027027, 21373101) and the Innovation Program of State Key Laboratory for Supramolecular Structure and Materials.

Keywords: calixarenes $\cdot$ enantioselective $\cdot \mathrm{HPV} \cdot$ inhibitors $\bullet$ L1 pentamer

\section{References:}

[1] B. Bishop, J. Dasgupta, X. S. Chen, Virol. J. 2007, 4, 3-10.

[2] J. Hernandez, A. Elahi, E. Siegel, D. Coppola, B. Riggs, D. Shibata, Am. J. Clin. Pathol. 2011, 135, $436-441$.

[3] X. Xie, Y. Liu, T. Zhang, Y. Xu, Q. Bao, X. Chen, H. Liu, X. Xu, Arch. Virol. 2013, 158, 193-199.

[4] H. H. Alphs, R. Gambhira, B. Karanam, J. N. Roberts, S. Jagu, J. T. Schiller, W. Zeng, D. C. Jackson, R. B. S. Roden, PNAS 2008,105, 5850-5855.

[5] T. J. Kemp, A. Hildesheim, M. Safaeian, J. G.Dauner, Y. Pan, C. Porras, J. T. Schiller, D. R. Lowy, R. Herrero, L. A. Pinto, Vaccine 2011, 29, 2011-2014. 
[6] S. Luciani, B. Jauregui, C. Kieny, J. K. Andrus. Immunotherapy 2009, 1, 795-807.

[7] D. R. Lowy, J. Clin. Invest. 2016, 126(1), 5-11.

[8] H. Faust, L. Toft, P. Sehr, M. Müller, J. Bonde, O. Forslund, L. Østergaard, M. Tolstrup, J. Dillner, Vaccine 2016, 34, 15591565.

[9] M. J. Conway, L. Cruz, S. Alam, N. D. Christensen, C. Meyers, PLoS ONE 2011,6(2), e16405.

[10] T. S. Baker, W. W. Newcomb, N. H. Olson, L. M. Cowsert, C. Olson, J. C. Brown, Biophys. J. 1991, 60, $1445-1456$.

[11] D.-D. Zheng, D. Pan, X. Zha, Y. Wu, C. Jiang and X. Yu, Chem. Commun. 2013, 49, 8546-8548.

[12] D.-D. Zheng, D.-Y. Fu, Y. Wu, Y.-L. Sun, L.-L. Tan, T. Zhou, S.-Q. Ma, X. Zha, Y.-W. Yang, Chem. Commun. 2014, 50, 32013203.

[13] J. K. Stanley, A. J. Ramirez, M. Mottaleb, C. K. Chambliss, B. W. Brooks, Environ. Toxicol. Chem. 2006, 25, $1780-1786$.

[14] F. Miao, J. Zhou, D. Tian, H. Li, Org. Lett. 2012, 14, 3572-3575.

[15] C. Han, X. Hou, H. Zhang, W. Guo, H. Li, L. Jiang, J. Am. Chem. Soc. 2011, 133, 7644-7647.

[16] C. S. Lim, J. Jankolovits, P. Zhao, J. W. Kampf, V. L. Pecoraro, Inorg. Chem. 2011, 50, 4832-4841.

[17] X. Mao, X. Jia, F. Qiu, Mol. Med. Rep. 2013, 8, 128-132.

[18] C. Moberg, Angew. Chem. Int. Ed. 2006, 45, 4721-4723.

[19] N. Morohashi, F. Narumi, N. Iki, T. Hattori, S. Miyano, Chem. Rev. 2006, 106, 5291-5316.

[20] Y.-S. Zheng, C. Zhang, Org. Lett. 2004, 6, 1189-1192.

[21] S. Cherenok, A. Vovk, I. Muravyova, A. Shivanyuk, V. Kukhar, J. Lipkowski, V. Kalchenko, Org. Lett. 2006, 8, 549-552.

[22] C.-Y. Goh, L. Baldini, A. Casnati, F. Jones, M. Mocerino, M. I. Ogden, F. Sansone, R. Ungaro, Supramol. Chem. 2014, 26, 488-499.

[23] M. Mourer, H. M. Dibama, S. Fontanay, M. Grare, R. E. Duval, C. Finance, J.-B. R.-d.-Vains, Bioorg. Med. Chem. 2009, 17, 5496-5509.

[24] D.-Y. Fu, S. Jin, D.-D Zheng, X. Zha, Y. Wu, ACS Med. Chem. Lett. 2015, 6, 381-385.

[25] S. Jin, D. Pan, X. Yu, Y. Wu, Y. Liu, F. Yin and X. S. Chen, Mol. BioSyst. 2014, 10, 724-727.

[26] T. Becker, C. Y. Goh, F. Jones, M. J. Mclldowie, M. Mocerino, M. I. Ogden, Chem. Commun. 2008, 3900-3902.

[27] D. K. Smith, Chem. Soc. Rev. 2009, 38, 684-694.

[28] C. Y. Goh, T. Becker, D. H. Brown, B. W. Skelton, F. Jones, M. Mocerino, M. I. Ogden, Chem. Commun. 2011, 47, $6057-6059$.

[29] A. R. Hirst, D. K. Smith, M. C. Feiters, H. P. M. Geurts, Chem. Eur. J. 2004, 10, 5901-5910.

[30] J. Zhang, D. -S. Guo, L. -H. Wang, Z. Wang, Y. Liu, Soft Matter 2011, 7, 1756-1762.

[31] M. Stanley, Gynecol. Oncol. 2010, 117, S5-S10.

[32] K. Wüthrich, NMR of Proteins and Nucleic Acids, Wiley-Interscience 1986, pp. 93-113.

[33] N. Gao, H. Sun, K. Dong, J. Ren, T. Duan, C. Xu, X. Qu, Nat. Commun. 2014, 5: 3422-3431. 